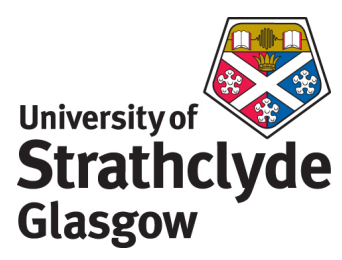

Graham, D. and Faulds, K. (2010) Mixed metal nanoparticle assembly and the effect on surface enhanced raman scattering. Nanoscale, 2 . pp. 78-80. ISSN 0306-0012

http://strathprints.strath.ac.uk/28837/

This is an author produced version of a paper published in Nanoscale, 2 . pp. 78-80.

ISSN 0306-0012. This version has been peer-reviewed but does not include the final publisher proof corrections, published layout or pagination.

Strathprints is designed to allow users to access the research output of the University of Strathclyde. Copyright $(C$ and Moral Rights for the papers on this site are retained by the individual authors and/or other copyright owners. You may not engage in further distribution of the material for any profitmaking activities or any commercial gain. You may freely distribute both the url (http://strathprints.strath.ac.uk) and the content of this paper for research or study, educational, or not-for-profit purposes without prior permission or charge. You may freely distribute the url (http://strathprints.strath.ac.uk) of the Strathprints website.

Any correspondence concerning this service should be sent to The Strathprints Administrator: eprints@cis.strath.ac.uk 


\title{
Mixed Metal Nanoparticle Assembly and the Effect on Surface Enhanced Raman Scattering
}

\author{
Fiona McKenzie, Karen Faulds and Duncan Graham* \\ Received (in $X X X, X X X)$ Xth $X X X X X X X X X 200 X$, Accepted $X t h X X X X X X X X X 200 X$ \\ ${ }_{5}$ First published on the web Xth $X X X X X X X X X 200 X$ \\ DOI: $10.1039 / \mathbf{b 0 0 0 0 0 0 x}$
}

\begin{abstract}
Here we report the assembly of mixed metal nanoparticles using an oligonucleotide-templated approach. Substitution of one of the gold nanoparticle probes with an analagous silver probe to 10 produce a hetero-metal duplex permitted surface enhanced Raman scattering of the dye label, exploiting the improved surface enhancement properties of silver nanoparticles whilst maintaining the surface chemistry benefits of gold nanoaprticles.
\end{abstract}

Surface enhanced Raman scattering (SERS) is a phenomenon 15 which has been investigated by a wide variety of techniques and employs the use of a range of surfaces to provide the surface enhancement component of the technique. One of the most favoured surfaces is that of metallic nanoparticles due to their ease of synthesis and handling in a variety of different 20 experimental formats. Individual nanoparticles will give rise to significant SERS signals when an appropriate SERS active molecule is immobilised on the surface. Larger signals can be obtained from these molecules by aggregating the nanoparticles into discrete clusters to improve the electromagnetic

${ }_{25}$ enhancement of the Raman tags and SERS can this be used to follow cluster formation. ${ }^{1,2}$ Aggregation can be achieved in a number of non specific methods involving alteration of surface charges and ultimately leads to precipitation of the nanoparticles. ${ }^{3-5}$ An alternative method to provide greater control

30 over the aggregation and assembly state of the nanoparticles is to use a DNA templated approach. This has previously been used to assemble nanoparticles in a number of different studies ${ }^{6-9}$ but only recently to investigate the enhancement of the SERS effect. $^{10,11}$ The main advantage to employing SERS for signal

35 transduction of the assembly process is the capability for identifying multiple assembly events simultaneously. The narrow and molecularly specific emssion features of SERS spectra make SERS significantly more favourable than other optical spectroscopies such as extinction or fluorescence.

40 To date, most of these studies have involved single metal nanoparticle assemblies, however, in order to probe the effectiveness of different types of metallic nanoparticles we have devised an approach to allow comparisons between the most commonly used metal nanoparticles, namely gold and silver.

45 This approach has also allowed us to demonstrate the phenomenon of using a nanoparticle purely as an enhancing species rather than containing a molecular probe of interest. Here we report the assembly of mixed metal nanoparticle conjugates induced by hybridisation of oligonucleotide nanoparticle ${ }_{50}$ conjugates to the complementary target sequence in a split probe assembly (figure 1).
One of the probes is based on a gold nanoparticle which in addition to being functionalised with oligonucleotides is also labelled with the strongly Raman active species, malachite green 55 isothiocyanate. The second probe incorporates a silver nanoparticle that is only functionalised with oligonucleotides and bears no SERRS active label. It is necessary to ensure that the probe to target concentration is in a 1:1 ratio, however since there are multiple DNA oligonucleotides per nanoparticle, the ${ }_{60}$ nanoparticle concentration needs to be adjusted accordingly. Furthermore, the number of oligonucleotides adsorbed onto 35 $\mathrm{nm}$ silver nanoparticles is approximately ten times greater than for $13 \mathrm{~nm}$ gold nanoparticles due to the increased surface area of silver compared with gold nanoparticles and so the gold ${ }_{65}$ nanoparticle concentration will be different to that of the silver nanoparticles. An approximate 1:1 ratio was achieved by using the gold nanoparticle probes, silver nanoparticle probes and target DNA at $0.5 \mathrm{nM}, 50 \mathrm{pM}$, and $50 \mathrm{nM}$, respectively.

The UV-visible spectra for the mixed metal assembly are 70 illustrated in figure 2. Before the addition of the target sequence, the plasmon resonance peak of the silver nanoparticles can be clearly identified at $410 \mathrm{~nm}$ exhibiting a shoulder at $520 \mathrm{~nm}$ which corresponds to the gold nanoparticles. Although the silver nanoparticle probes are used at a lower concentration, their larger 75 extinction coefficient makes the silver peak most dominant. Addition of the target and subsequent hybridisation to the probes resulted in aggregation of the nanoparticles. This gives rise to a red-shift and broadening of the plasmon resonances due to the change in dielectric experienced by each nanoparticle. ${ }^{12}$

80 Upon aggregation, the electromagnetic fields surrounding each nanoparticle couple to produce 'hot spots'. These are regions of high electromagnetic field intensity and are responsible for large surface enhancement effects of the Raman signals. ${ }^{13}$ Furthermore, the plasmon resonance wavelength of the 85 nanoparticles is tuned to that of the laser excitation promoting plasmon excitation and improved surface enhancement effects. Since the addition of the DNA target results in nanoparticle aggregation, it can be used to generate increased SERRS intensities. This is illustrated in figure 3. The SERRS intensity of 90 the mixed metal assembly is more than a factor of 3 greater than the monodisperse nanoparticles in absence of the target sequence. Oligonucleotide-functionalised gold nanoparticles have been shown to yield optimum SERRS spectra at longer excitation wavelengths due to the red-shifted plasmon resonance 95 wavelength compared with silver. It has been suggested that electronic transitions associated with the bulk metal which occur at shorter wavelengths than the plasmon excitation peak are 
responsible for the poor behaviour of gold nanoparticle based analyses at $514.5 \mathrm{~nm} .{ }^{14}$ As such, the mixed metal assembly was analysed at $632.8 \mathrm{~nm}$ and the SERRS label was chosen so that it was in resonance with this excitation wavelength.

To demonstrate that the observed increase in SERRS intensity is the result of specific Watson-Crick hydrogen bonding, a non-complementary target was added to the nanoparticle probes. Only a slight increase was observed which is likely to be the result of a few base pairing interactions, of which the most 10 energetically favourable are shown in figure $1 b$.

Silver nanoparticles are known to yield significantly greater surface enhancement of Raman signals than gold nanoparticles, ${ }^{14}$ although it is advantageous to utilise gold nanoparticles due to the more established surface chemistry which often exploits the high 15 affinity thiols have for gold surfaces. Gold nanoparticles are renowned for having improved stability compared with silver and so are often preferred for use in solution-based experiments. We have found that surface modification of silver nanoparticles for labelling purposes often resulted in irreversible aggregation as a 20 result of the hydrophobic nature of many commercially available fluorophores. A mixed metal assembly profits from the greater stability of gold nanoparticles and the exceptional enhancement effects provided by the silver nanoparticles. The requirement for using silver is emphasised in figure 4 which presents the spectra 25 when the silver nanoparticle probe is substituted so that the assembly consists of two gold nanoparticle probes. Although a colour change from red to blue was observed and there was a significant shift in the plasmon resonance, the increase in SERRS intensity was negligible. This indicates that the plasmon 30 resonance coupling is not as effective as for the gold-silver scenario, or indeed a silver-silver scenario. ${ }^{10}$ This is a highly significant result as it proves that silver has a larger enhancing capacity than gold and provides evidence that in terms of electromagnetic enhancement of SERS, silver is the optimal 35 metal to use.

\section{Conclusions}

In conclusion, we have shown that we can study the effect of 40 different types of metal nanoparticles on an assembly based enhanced SERS effect using oligonucleotide templating. Silver nanoparticles provide greater enhancement compared to gold nanoparticles and this has also allowed us to demonstrate that only one species needs to be modified to contain the Raman 45 active tag rather than all species as in previous studies. This is a significant step forward in the understanding of assembly processes of nanoparticles in relation to surface enhanced Raman scattering and offers significant opportunities for exploitation in terms of using this data to better control and understand so nanoparticle assemblies and advanced optical spectroscopies.

\section{Notes and references}

Centre for Molecular Nanometrology, Pure and Applied Chemistry, University of Strathclyde, 295 Cathedral Street, Glasgow, G1 IXL, UK E-mail: Duncan.Graham@strath.ac.uk

$55 \dagger$ Electronic Supplementary Information (ESI) available: experimental. See DOI: $10.1039 / \mathrm{b} 000000 \mathrm{x} /$
1. I. Khan, D. Cunningham, R. E. Littleford, D. Graham, W. E. Smith and D. W. McComb, Anal. Chem., 2006, 78, 224-230.

602 K. Faulds, R. E. Littleford, D. Graham, G. Dent and W. E. Smith, Anal. Chem., 2004, 76, 592-598.

3. J. C. Jones, C. McLaughlin, D. Littlejohn, D. A. Sadler, D. Graham and W. E. Smith, Anal. Chem., 1999, 71, 596-601.

$4 . \quad$ C. H. Munro, W. E. Smith, M. Garner, J. Clarkson and P. C. White, Langmuir, 1995, 11, 3712-3720.

5. D. Cunningham, R. E. Littleford, W. E. Smith, P. J. Lundahl, I. Khan, D. W. McComb, D. Graham and N. Laforest, Faraday Discuss., 2006, 132, 135-145.

6. C. A. Mirkin, R. L. Letsinger, R. C. Mucic and J. J. Storhoff, $70 \quad$ Nature, 1996, 382, 607-609.

7. A. P. Alivisatos, K. P. Johnsson, X. G. Peng, T. E. Wilson, C. J. Loweth, M. P. Bruchez and P. G. Schultz, Nature, 1996, 382, 609-611.

8. C. J. Loweth, W. B. Caldwell, X. H. Peng and A. P. Alivisatos, 75 Angew. Chem. Int. Edit., 1999, 38, 1808-1812.

$9 . \quad$ R. C. Jin, G. S. Wu, Z. Li, C. A. Mirkin and G. C. Schatz, J. Am. Chem. Soc., 2003, 125, 1643-1654.

$10 . \quad$ D. Graham, D. G. Thompson, W. E. Smith and K. Faulds, Nat. Nanotech., 2008, 3, 548 - 551

80 11. X. Qian, X. Zhou and S. Nie, J. Am. Chem. Soc., 2008, 130, 14934-14935.

12. D. G. Thompson, R. J. Stokes, R. W. Martin, P. J. Lundahl, K. Faulds and D. Graham, Small, 2008, 4, 1054-1057.

13. J. M. McMahon, A.-I. Henry, K. L. Wustholz, M. J. Natan, R. 85 G. Freeman, R. P. Van Duyne and G. C. Schatz, Anal. Bioanal. Chem., 2009, advance article, DOI 10.1007/s0021600009-02738-00214.

14. R. J. Stokes, A. Macaskill, P. J. Lundahl, W. E. Smith, K. Faulds and D. Graham, Small, 2007, 3, 1593-1601. 
a)

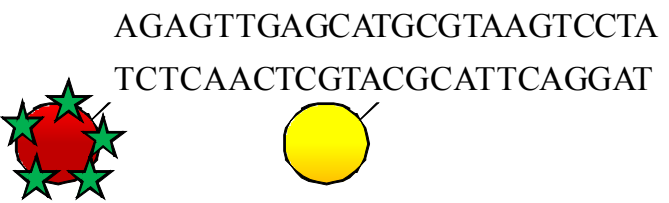

b)

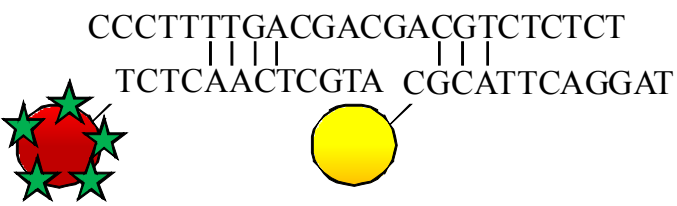

c)

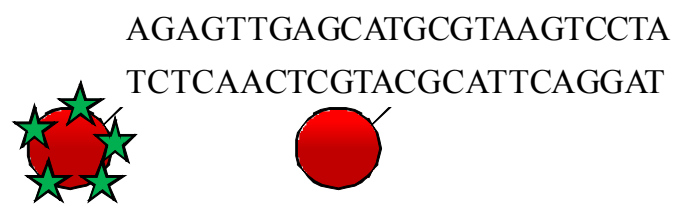

${ }_{5}$ Figure 1. Head-to-tail assembly of oligonucleotide probes: a) mixed metal nanoparticle assembly; b) mixed metal probes in presence of non-complementary DNA; c) gold nanoparticle assembly. The red and yellow spheres represent gold and silver nanoparticles, respectively. The green stars represent malachite green isothiocyanate.

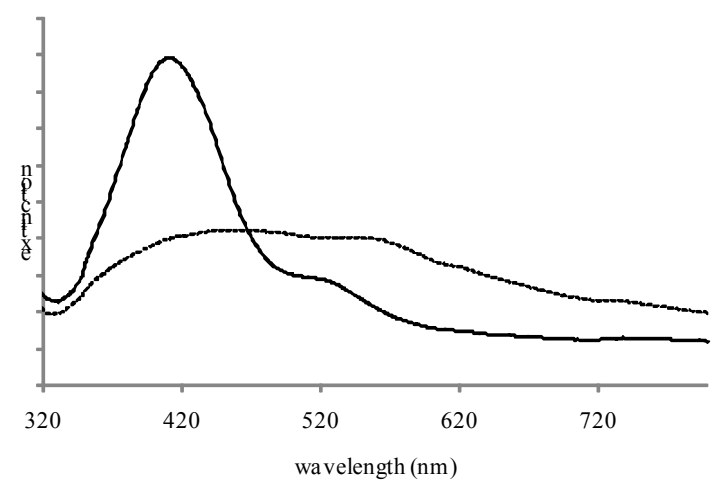

${ }^{10}$ Figure 2. Extinction spectra of mixed metal probes before (solid line) and after (dashed line) the addition of the complementary target sequence.
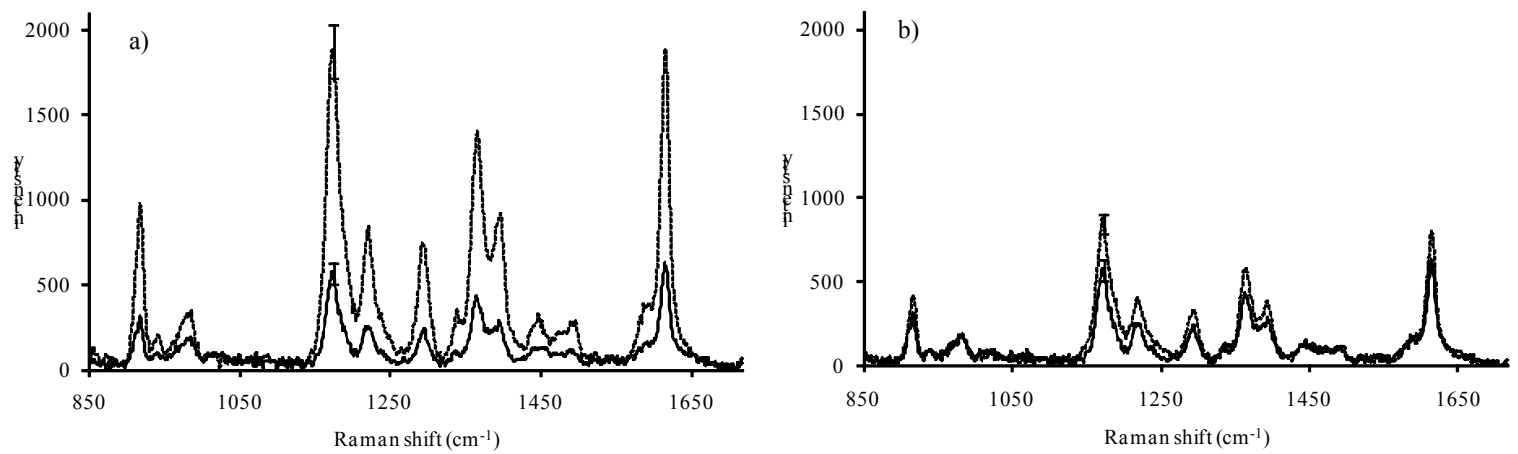
Figure 3. SERRS before (solid line) and after (dashed line) the addition of a) the complementary and b) a non-complementary target sequence to the mixed metal probes using a $632.8 \mathrm{~nm}$ excitation wavelength.

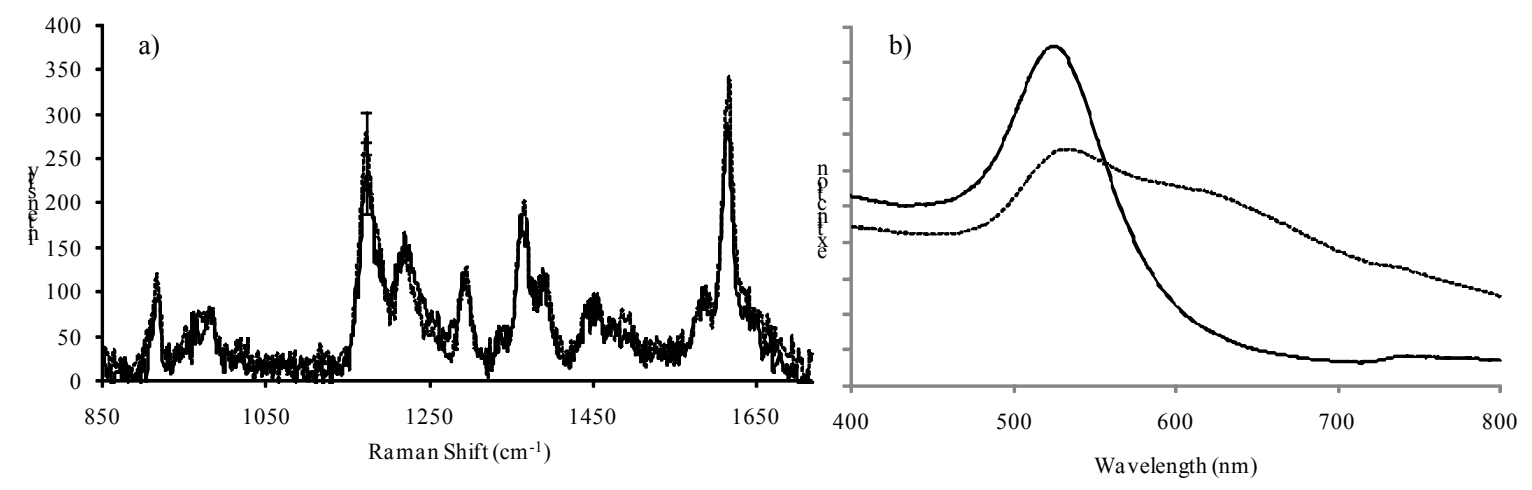

Figure 4. SERRS (a) and UV-visible (b) spectra of the gold nanoparticle assembly before (solid line) and after (dashed line) the addition 10 of the target DNA. The SERRS spectra were recorded using a $632.8 \mathrm{~nm}$ excitation wavelength. 


\section{ESI}

\section{Experimental Section}

DNA oligonucleotides were purchased from ATD Bio, Southampton and malachite green isothiocyanate was purchased from Invitrogen, 5 Paisley.

Thiol modified DNA (10 nmoles): 5' SH (HEG) $)_{3}$ TCT CAA CTC GTA 3' and 5' SH (HEG) $)_{3}$ CGC ATT CAG GAT 3' was added to $13 \mathrm{~nm}$ citrate-reduced gold nanoparticles $(1 \mathrm{~mL}, 4.5 \mathrm{nM})$ and $35 \mathrm{~nm}$ silver nanoparticles $(1 \mathrm{~mL}, 0.4 \mathrm{nM})$, respectively, and salt-aged as previously reported. ${ }^{1}$ Nanoparticle conjugate concentrations were evaluated using extinction spectroscopy $\left(\varepsilon=2.87 \times 10^{10} \mathrm{M}^{-1} \mathrm{~cm}^{-1}\right.$ at $400 \mathrm{~nm}$ for silver ${ }^{2}$ and $\varepsilon=2.7 \times 10^{8} \mathrm{M}^{-1} \mathrm{~cm}^{-1}$ at $520 \mathrm{~nm}$ for gold $\left.{ }^{3}\right)$.

10 Malachite green isothiocyanate, MG ITC, $(500 \mu \mathrm{L}, 1 \mu \mathrm{M})$ was added to the oligonucleotide gold nanoparticle conjugate $(500 \mu \mathrm{L}, 6.7$ $\mathrm{nM}$ ) and incubated for 16 hours in the dark, followed by centrifugation and resuspension in $0.3 \mathrm{M}$ PBS. The nanoparticle assembly was constructed by hybridisation of the target sequence: 5' ATC CTG AAT GCG TAC GAG TTG AGA 3' (50 nM) with the MG ITC labelled oligonucleotide functionalised gold conjugate $(0.5 \mathrm{nM})$ and the oligonucleotide silver conjugate $(50 \mathrm{pM})$.

SERRS spectra were recorded 5 minutes after the addition of the target sequence using a Renishaw Probe Spectrometer with a 514.5 $15 \mathrm{~nm}$ argon ion laser. UV-visible spectra were recorded using a Varian Cary Bio 300 spectrometer.

1. McKenzie, F.; Faulds, K.; Graham, D. Small 2007, 3, 1866-1168.

2. Yguerabide, J.; Yguerabide, E. E. Anal. Biochem. 1998, 262, 137-156.

3. Jin, R. C.; Wu, G. S.; Li, Z.; Mirkin, C. A.; Schatz, G. C. J. Am. Chem. Soc. 2003, 125, 1643-1654. 